\title{
FINANÇAS COMPORTAMENTAIS DE ESTUDANTES \\ UNIVERSITÁRIOS: UMA ANÁLISE MULTIVARIADA
}

\author{
Luis Felipe Dias Lopes ${ }^{1}$ \\ Steffani Nikoli Dapper ${ }^{2}$ \\ Claudia Lunardi ${ }^{3}$ \\ Tuani de Oliveira Tavares ${ }^{4}$
}

\begin{abstract}
Resumo
O dinheiro é um conceito estudado por diferentes áreas da ciência e está relacionado à vida cotidiana das pessoas. O objetivo desta pesquisa consistiu em identificar os fatores que representam o significado do dinheiro para jovens, que se mudaram para a cidade de Santa Maria/RS, em virtude da oportunidade de estudar na Universidade Federal de Santa Maria (UFSM), e assim se encontram em maior contato com as finanças pessoais, devido à distância de seus familiares. $\mathrm{O}$ presente estudo se caracteriza como uma pesquisa descritiva e quantitativa. A amostra foi não probabilística por conveniência, sendo composta por 499 estudantes de graduação de diversos cursos. Como instrumento para coleta dos dados, foi utilizada a Escala de Significado do dinheiro de Schwartz (1992), adaptada por Moreira (2000). A fim de identificar os fatores que representam o significado do dinheiro, foi realizada uma análise fatorial exploratória. Esta técnica multivariada permitiu expressar, o conjunto inicial de 82 variáveis, em apenas 10 fatores, compostos por 48 questões que permaneceram na análise. Os dois fatores mais relevantes, denominados de Conflito e de Poder, compreenderam variáveis que abordam questões relacionadas aos problemas que o dinheiro pode causar, como desavenças, desarmonia, traições e frustrações, bem como ao poder e privilégio social associado a posse do dinheiro como, por exemplo, a ascensão social que este permite, a autoridade, a dominação e o prestígio. Em suma, acredita-se que o dinheiro possui significados que vão muito além de sua função principal, que seria a função de troca.
\end{abstract}

Palavras-chave: Dinheiro. Finanças Comportamentais. Estudantes.

\section{BEHAVIORAL FINANCE OF UNIVERSITY STUDENTS:}

\footnotetext{
${ }^{1}$ Doutor da Universidade Federal de Santa Maria - UFSM

${ }^{2}$ Mestre em Engenharia de Produção - UFSM

${ }^{3}$ Mestre em Administração - UFSM

${ }^{4}$ Graduada em Ciências Econômicas - UFSM
} 


\title{
A MULTIVARIATE ANALYSIS
}

\begin{abstract}
Money is a concept studied by different areas of science and is related to people's daily lives. The objective of this research is to identify the factors that represent the meaning of money for young people, who moved to the city of Santa Maria / RS, due to the opportunity to study at the Federal University of Santa Maria (UFSM). Goal In greater contact with how finance, please distance your family members. The present study is characterized as a descriptive and quantitative research. A non-probabilistic sample for convenience, being composed of 499 undergraduate students of several courses. As a tool for data collection, it was used in the Schwartz Money Scale (1992), adapted by Moreira (2000). In order to identify the factors that represent the meaning of money, an exploratory factorial analysis was performed. This multivariate technique allowed us to express the initial set of 82 variables in only 10 factors, composed of 48 questions that maintain the analysis. Problems, knowledge, and power, as well as the power and social privilege associated with the possession of money, such as disagreements, disharmony, betrayals and frustrations, as well as the associated power and social privilege. Allows, an authority, a domination and prestige. In sum, money is believed to have meanings that go far beyond its primary function, which is a function of exchange.
\end{abstract}

Keywords: Money; Behavioral Finance; Students.

\section{Resumen}

El dinero es un concepto estudiado por diferentes áreas de la ciencia y está relacionado con la vida cotidiana de las personas. El objetivo de esta investigación consistió en identificar los factores que representan el significado del dinero para jóvenes, que se trasladaron a la ciudad de Santa Maria / RS, en virtud de la oportunidad de estudiar en la Universidad Federal de Santa María (UFSM), y así se encuentran en mayor contacto con las finanzas personales, debido a la distancia de sus familiares. El presente estudio se caracteriza como una investigación descriptiva y cuantitativa. La muestra no fue probabilística por conveniencia, siendo compuesta por 499 estudiantes de graduación de diversos cursos. Como instrumento para la recolección de datos, se utilizó la Escala de Significado del dinero de Schwartz (1992), adaptada por Moreira (2000). A fin de identificar los factores que representan el significado del dinero, se realizó un análisis factorial exploratorio. Esta técnica multivariada permitió expresar, el conjunto inicial de 82 variables, en apenas 10 factores, compuestos por 48 cuestiones que permanecieron en el análisis. Los dos factores más relevantes, denominados de Conflicto y de Poder, comprendieron variables que abordan cuestiones relacionadas con los problemas que el dinero puede causar, como desavenencias, desarmonía, traiciones y frustraciones, así como al poder y privilegio social asociado a la posesión del dinero como, por ejemplo, el ascenso social que éste permite, la autoridad, la dominación y el prestigio. En suma, se cree que el dinero tiene significados que van mucho más allá de su función principal, que sería la función de cambio. 
Palabras clave: Dinero. Finanzas Comportamentales. Estudiantes.

\section{INTRODUÇÃO}

Finanças Comportamentais é uma área de pesquisa que vem ganhando crescente reconhecimento no mundo acadêmico e fora dele. Seu traço distintivo é a incorporação de conceitos de outras áreas, como os da psicologia e os da sociologia, para as áreas da economia e da administração, a fim de explicar as decisões financeiras dos indivíduos. Este campo de estudo foi desenvolvido por dois psicólogos israelenses, Daniel Kahneman e Amos Tversky, em 1979, por meio de um trabalho intitulado "Teoria do Prospecto" (Arruda, 2006).

As teorias tradicionais de Finanças foram construídas a partir de uma abordagem microeconômica neoclássica, cujo paradigma central é a racionalidade dos agentes econômicos, entretanto, a validade das premissas assumidas por essas teorias vem sendo questionada e uma de suas abordagens é o significado que as pessoas dão ao dinheiro.

O dinheiro é um conceito estudado por diferentes áreas da ciência e está relacionado à vida cotidiana das pessoas. De maneira significativa, a psicologia fornece subsídios para a administração financeira chegar ao entendimento da relação do homem com o dinheiro, em particular, aos pesquisadores que desenvolvem instrumentos capazes de mensurar vários aspectos dessa relação.

A conversibilidade que o dinheiro possibilita, faz dele um objeto concomitantemente universal, presente em todos os momentos da vida econômica cotidiana, constituindo parte significativa da vida social (Moreira, 2000). No entanto, o dinheiro, que deveria ter o papel de ser um intermediário de trocas, acaba assumindo diversos papeis, visto que as moedas refletem a mentalidade de um povo. Por meio dela, podem ser observados aspectos políticos, econômicos, tecnológicos e culturais.

Frente a isto, algumas pesquisas surgiram para identificar o significado do dinheiro, buscando delimitar a estrutura cognitiva deste construto e variáveis a ele relacionadas (Moreira, 2000). Estes estudos têm fomentado a denominada psicologia econômica que visa compreender como os fenômenos econômicos afetam a vida dos indivíduos e, por sua vez, como o comportamento destes pode influenciar na economia. 
Desta forma, o entendimento das relações entre os indivíduos e o dinheiro, forma um campo de estudo emergente, tanto na psicologia como na administração e na economia.

Diante deste cenário, o objetivo desta pesquisa consistiu em identificar os fatores que representam o significado do dinheiro para jovens, que se mudaram para a cidade de Santa Maria/RS, em virtude da oportunidade de estudar na Universidade Federal de Santa Maria (UFSM). Buscou-se compreender, por meio de análises de técnicas multivariadas, o comportamento financeiro destes estudantes, que ao se mudarem de suas casas, iniciam um relacionamento mais próximo com o controle das suas finanças pessoais, ocasionado pela distância de seus familiares.

\section{FINANÇAS COMPORTAMENTAIS}

Os estudos de Loewenstein (1996), já indicavam no final do século XIX, que para que o comportamento do consumidor fosse completamente entendido, era necessário reconhecer que os indivíduos são influenciados por preocupações racionais de longo prazo, bem como por fatores emocionais de curto prazo, ou seja, existe inconsistência temporal na preferência do consumidor.

A área de Finanças Comportamentais é muito recente, onde se tem muito a explorar, pois surgiu há aproximadamente quarenta anos atrás, por meio das pesquisas realizadas pelos psicólogos Amos Tversky e Daniel Kahneman (1974). Segundo Macedo Jr. (2003), estes dois pesquisadores, que testavam a racionalidade dos tomadores de decisão, criticavam a teoria da racionalidade. Tversky e Kahneman (1979) identificaram várias situações em que o ser humano tomava decisões baseadas em vieses que os afastavam da racionalidade, o que contrariava os pressupostos desta moderna teoria.

A teoria da racionalidade tem como eixo central basear-se em uma visão do consumidor apoiado na Racionalidade Econômica, isto é, o comportamento do consumidor obedece a um padrão egoísta e maximizador, cujas escolhas de consumo são pautadas por uma busca do maior benefício (prazer ou satisfação), ao menor custo possível (desconforto ou sofrimento). Por ser uma abordagem teórica, calcada no

utilitarismo psicológico, o comportamento de consumo tem por horizonte a maximização da utilidade, isto é, os esforços de escolha de um consumidor sempre teriam como base a maximização dos graus de satisfação psicológica e do prazer obtido com o uso dos produtos e serviços adquiridos (Tversky; Kahnemann, 1979). 
Os trabalhos de Tversky e Kahneman em 1974 e 1979 formaram a base teórica para a análise do comportamento dos investidores e, posteriormente, foram complementados por Werner De Bont e Richar Tahler, em seu trabalho intitulado Does the Stock Market Overreact. Neste artigo, os autores reagiram às falhas do modelo tradicional e fizeram uma aplicação da heurística da representatividade de Tversky e Kahneman (1974) na precificação do mercado e constataram que os investidores sobre reagem tanto às boas, como às más notícias.

O autor Simon (1957) sugere que o julgamento individual do indivíduo se restringe pela sua racionalidade, isto é, apesar dos indivíduos tentarem tomar decisões racionais, a simplificação ou a falta de informações importantes, ou ainda, a falta dos critérios relevantes para a solução dos problemas, limitações de inteligência e de percepção, restrições de tempo e de custos, e limitações na capacidade de retenção das informações na memória humana, impedem que os indivíduos tomem decisões ótimas ou maximizadas.

Este autor explicitou sua apreensão relacionada com a adequação à realidade das premissas implícitas na abordagem clássica da teoria da decisão no campo da administração, em especial, sobre o pressuposto da racionalidade e do comportamento maximizador da Teoria da Utilidade Esperada (TUE), afirmando que a moderna teoria não oferece nenhuma característica psicológica ao comportamento humano, o que é evidenciado pela limitada capacidade descritiva e preditiva desta teoria (Simon, 1979).

Como os modelos que consideravam os indivíduos totalmente racionais pareciam não responder integralmente às questões enfrentadas pelos gestores no mundo real, o estudo do comportamento humano começou a tornar-se mais importante, e com ele o conceito de racionalidade limitada, então, nos anos 90, foram introduzidos conceitos de psicologia para relacioná-los ao mercado financeiro, desenvolvendo-se o campo de finanças comportamentais.

Em suma, finanças comportamentais é um novo entendimento sobre os mercados financeiros que emergiu, em parte, como uma resposta às dificuldades encontradas pelos paradigmas tradicionais. Este campo de estudo, postula que alguns fenômenos financeiros são mais bem entendidos, usando modelos nos quais os agentes não são completamente racionais e seus estudos têm se concentrado na compreensão das ilusões cognitivas e de suas implicações no comportamento dos tomadores de decisão. Assim como, em identificar como tais ilusões cognitivas podem influenciar o mercado financeiro. 
Um importante componente do estudo do comportamento, na literatura financeira, analisa o processo decisório de todos os tipos de investidores (especialistas, investidores profissionais, estudantes e o público em geral), em termos de temas específicos (excesso de confiança ou aversão à perda, por exemplo), sendo que neste sentido, estudiosos das Finanças Comportamentais têm um diferente ponto de vista sobre o risco e tomada de decisão, quando comparado às Finanças Tradicionais.

Sortino (2001) destaca que, recentes pesquisas na área das Finanças Comportamentais, alegam que os investidores não procuram o maior retorno para um determinado nível de risco, como a teoria tradicional propõe, em vez de maximizar o valor esperado preferem atender aos seus valores psicológicos (aversão à perda ou propensão ao risco).

O ser humano, para a área de Finanças Comportamentais, não é totalmente racional, mas sim um homem simplesmente normal. Essa normalidade implica em um ser que age, frequentemente, de maneira irracional e que tem suas decisões influenciadas por emoções e por erros cognitivos, fazendo com que ele entenda um mesmo problema de formas diferentes, dependendo da maneira como é analisado (Halfeld; Torres, 2001).

Partindo deste pressuposto, o campo das Finanças Comportamentais faz parte deste esforço de reformulação do paradigma vigente e busca explicar, de maneira satisfatória, uma gama de fenômenos regularmente observados nos mercados financeiros e incompatíveis com as predições dos modelos tradicionais.

Nofsinger (2006) afirma que nesses estudos o foco está nos aspectos comportamentais das decisões financeiras, na forma como são tomadas e formuladas as decisões, como se forma a visão dos seres humanos com relação ao dinheiro e com tudo que se relaciona a ele.

Referente à visão que os jovens têm sobre o dinheiro Zerrenner (2007) recomenda a educação financeira, o instrumento de coleta de dados, população e amostra, que se constitui como sendo uma ferramenta que auxilia a mudar as preferências do fazedor, monitorando o seu comportamento, alterando incentivos e modificando regras.

\section{MATERIAIS E MÉTODOS}


O presente estudo é classificado como quantitativo, do tipo survey, e se caracteriza como uma pesquisa descritiva que, de acordo com Vergara (2000), esta estabelece as relações entre variáveis, isto é, aquelas que visam estudar as características de um grupo: sua distribuição por idade, sexo, procedência, nível de escolaridade, estado de saúde física e mental, entre outros aspectos. Procura descobrir, com a precisão possível, a frequência com que um fenômeno ocorre, sua relação e conexão com os outros, sua natureza e características.

Para a coleta de dados optou-se pela utilização de um questionário constituído de duas partes. A primeira buscou identificar aspectos relacionados ao perfil da amostra investigada. A segunda compreendeu questões relativas ao tema pesquisado, com base na Escala de Significado do dinheiro, elaborada por Schwartz (1992) e adaptada por Moreira (2000). O questionário proposto por Moreira (2000) é composto por 200 questões. Para a realização deste estudo, optou-se por utilizar 82 questões capazes de atingir o objetivo proposto por esta pesquisa. As questões são avaliadas por uma escala do tipo Likert que varia de (1) discordo totalmente a (5) concordo totalmente.

A população da pesquisa se refere a estudantes de todos os cursos de graduação da UFSM, que se mudaram para a cidade de Santa Maria em virtude da opção de ingressar em um curso superior. A amostragem foi não probabilística, por conveniência.

Hair Jr. et al. (2005) destacam, como regra geral, que para a realização da análise fatorial, se deve ter pelo menos cinco vezes mais observações do que o número de variáveis a serem analisadas. Assim, considerando-se que o instrumento foi composto por 82 questões, estipulou-se um número mínimo de 410 respondentes, sendo obtidos um total 499 questionários respondidos.

A coleta dos dados se deu nas áreas externas aos prédios da UFSM, fora das salas de aula, nas calçadas do campus, paradas de ônibus, entradas de prédios, bibliotecas, áreas externas aos Restaurantes Universitários, ou seja, nos momentos livres dos estudantes. Os alunos que estiveram nesses locais foram abordados, recebendo a explicação da pesquisa.

Após a coleta dos dados, estes foram tabulados em uma planilha do Software Microsoft Excel, e analisados por meio do software Statistical Package for the Social Sciences (SPSS), versão 18.0.

Os dados referentes ao perfil dos estudantes foram analisados a partir de estatísticas descritivas (frequências absoluta e relativa). 
A fim de verificar os fatores que correspondem ao significado do dinheiro para os estudantes que vão residir em Santa Maria, foi realizada uma análise fatorial exploratória que, segundo Hair Jr. et al. (2009), aborda o problema de como analisar a estrutura de inter-relações (covariâncias) existentes entre as variáveis, com a definição de uma série de dimensões subjacentes comuns, conhecidas como fatores.

Dentre os métodos existentes para a extração dos fatores, destacam-se o método dos componentes principais e o da máxima verossimilhança. No presente estudo, foi utilizado o método de componentes principais, pois de acordo com Malhotra (2001), este é o mais recomendado quando a preocupação principal do pesquisador é determinar o número mínimo de fatores que correspondem a variância máxima dos dados.

Para averiguar se a análise de componentes principais (ACP) é adequada ao conjunto de dados obtidos e, para aferir a qualidade das correlações entre as variáveis e dar continuidade a análise fatorial, a ponto de apenas alguns fatores serem capazes de representar grande parte da variabilidade dos dados, foi aplicado o teste de esfericidade de Bartlett. O teste Kaiser-Meyer-Olkin (KMO) foi utilizado para verificar se a correlação entre cada par de variáveis pode ser explicada pelas demais variáveis incluídas no estudo. O KMO é um teste estatístico que pode assumir valores entre zero e um, em que valores menores que 0,5 indicam que a análise fatorial não é satisfatória (Pestana; Gageiro, 2003).

É possível encontrar tantos fatores quantas forem as variáveis pesquisadas. Entretanto, os pesquisadores deste estudo buscaram resumir as informações contidas nas variáveis originais, em um número menor de fatores. Para isso, foi preciso definir entre os métodos disponíveis, qual seria o critério de determinação do número de fatores a serem utilizados. Conforme Hair Jr.et al. (2005), cada variável contribui com um valor correspondente a "1" do autovalor total. Assim, apenas os fatores que têm raízes latentes ou, autovalores maiores do que 1, são considerados significativos.

Neste estudo foi utilizada a rotação Varimax normalizada como medida para rotação dos fatores. Para avaliar a confiabilidade dos fatores obtidos, após a análise fatorial, foi utilizado o Alfade Cronbach, cuja medida varia de 0 a 1 e, valores como 0,60 são considerados o limite inferior de aceitabilidade. A confiabilidade representa o grau em que uma variável ou conjunto de variáveis é consistente com o que se pretende medir (Hair Jr. et al., 2005). 
Após a Análise Fatorial, foram observados os fatores tendo por base as cargas fatoriais, a partir da recomendação de Hair Jr. et al. (2005), considerando-se satisfatória a carga fatorial superior a 0,3 .

O presente estudo foi registrado junto ao Gabinete de Projetos (GAP) do Centro de Ciências Sociais e Humanas (CCSH) da Universidade Federal de Santa Maria (UFSM) sob número 027246, e posteriormente foi encaminhado e aprovado pelo Comitê de Ética em Pesquisa (CEP-Reitoria/UFSM) cujo Certificado de Apresentação para Apreciação Ética (CAAE) é 0131.0.243.000-11.

Atendendo às Diretrizes e Normas Regulamentadoras de Pesquisas Envolvendo Seres Humanos (Resolução CNS 196/96), foram disponibilizados aos participantes o Termo de Consentimento Livre Esclarecido (TCLE), que foi assinado, após explicação completa e pormenorizada por parte dos pesquisadores, sobre a natureza da pesquisa, seus objetivos, métodos, benefícios previstos, potenciais riscos e o incômodo que esta poderia acarretar, formulada no termo de consentimento, autorizando sua participação voluntária na pesquisa.

Além disso, os autores desta pesquisa tiveram o compromisso com a privacidade e a confidencialidade dos dados utilizados, preservando integralmente o anonimato dos participantes.

\section{ANÁLISE DOS RESULTADOS}

Neste item serão explanados os resultados referentes ao perfil dos respondentes, bem como a análise fatorial exploratória, a qual permitiu a identificação dos fatores que representam o significado do dinheiro para os estudantes.

\subsection{PERFIL DOS RESPONDENTES}

Na Tabela 1 é possível observar os resultados referentes ao perfil dos estudantes que participaram da amostra desta pesquisa sendo que, dentre esses, 52,71\% possuem idade entre 18 e 39 anos, com idade média de 21 anos. 
Tabela 1 - Perfil dos estudantes

\begin{tabular}{|c|c|c|c|}
\hline \multicolumn{2}{|l|}{ Variáveis } & \multirow{2}{*}{$\begin{array}{c}\begin{array}{c}\text { Frequência } \\
\text { acumulada }\end{array} \\
263\end{array}$} & \multirow{2}{*}{$\begin{array}{c}\begin{array}{c}\text { Frequência } \\
\text { relativa }\end{array} \\
52,71 \%\end{array}$} \\
\hline & Feminino & & \\
\hline Genero & Masculino & 236 & $47,29 \%$ \\
\hline \multirow{12}{*}{ Estado ou País de naturalidade } & Argentina & 1 & $0,20 \%$ \\
\hline & Ceará & 1 & $0,20 \%$ \\
\hline & Goiás & 1 & $0,20 \%$ \\
\hline & Minas Gerais & 2 & $0,40 \%$ \\
\hline & Mato Grosso & 1 & $0,20 \%$ \\
\hline & Pará & 1 & $0,20 \%$ \\
\hline & Pernambuco & 1 & $0,20 \%$ \\
\hline & Paraná & 2 & $0,40 \%$ \\
\hline & Rio de Janeiro & 5 & $1 \%$ \\
\hline & Rio Grande do Sul & 461 & $92,38 \%$ \\
\hline & Santa Catarina & 21 & $4,21 \%$ \\
\hline & São Paulo & 2 & $0,40 \%$ \\
\hline \multirow{3}{*}{ Estado Civil } & Casado (a) & 16 & $3,21 \%$ \\
\hline & Solteiro (a) & 482 & $96,59 \%$ \\
\hline & Separado (a) & 1 & $0,20 \%$ \\
\hline \multirow{2}{*}{ Filhos } & Não & 493 & $98,80 \%$ \\
\hline & Sim & 6 & $1,20 \%$ \\
\hline \multirow{6}{*}{ Moradia } & Própria & 58 & $11,62 \%$ \\
\hline & Alugada & 211 & $42,28 \%$ \\
\hline & Casa do Estudante & 184 & $36,87 \%$ \\
\hline & Com Parentes & 25 & $5,01 \%$ \\
\hline & Pensão & 7 & $1,40 \%$ \\
\hline & Outros & 14 & $2,81 \%$ \\
\hline
\end{tabular}

Em relação à cidade de origem, 92,38\% são naturais de cidades do Estado do

Rio Grande do Sul, sendo encontrada uma variedade de 183 municípios e um estudante da Argentina. Há também uma predominância de respondentes solteiros (96,59\%). No que tange ao número de filhos, somente 6 possuem filhos. A grande parte dos estudantes $(42,28 \%)$ paga aluguel, seguido pelos respondentes que residem na casa do estudante $(36,87 \%)$. Uma minoria de $1,4 \%$, ou seja, 7 pessoas afirmaram residir em pensões.

Os estudantes foram questionados quanto ao seu comportamento em relação aos gastos realizados, bem como quanto a frequência em que poupam e a interação realizada com o dinheiro. Pode-se verificar, a partir da Tabela 2, que a maioria dos respondentes, $47,9 \%$, afirmaram gastar o mesmo que ganham, enquanto $16,83 \%$ relataram gastar mais da quantidade que recebem como renda. No entanto, quando questionados sobre o ato 
de poupar dinheiro, apenas 9,02\% afirmam poupar sempre, 34,67\% poupam frequentemente, $32,67 \%$ poupam somente algumas vezes, enquanto $6,01 \%$ nunca poupam dinheiro.

Com relação à responsabilidade financeira dos estudantes, no que se refere a maneira como administravam o dinheiro antes de mudarem para Santa Maria, 39,68\% afirmaram que já possuíam responsabilidades como, pagar contas e controlar o seu dinheiro, 29,06\% não tinham preocupações relacionadas a isso, 26,65\% recebiam mesada para seus gastos pessoais e controlavam este dinheiro. Somente $4,61 \%$ não recebiam mesada, porém acabavam precisando de dinheiro posteriormente.

Tabela 2 - Características dos alunos quanto às responsabilidades relacionadas ao dinheiro

\begin{tabular}{|c|c|c|c|}
\hline Variáveis & Alternativas & $\begin{array}{c}\text { Frequência } \\
\text { absoluta }\end{array}$ & $\begin{array}{c}\text { Frequência } \\
\text { relativa }\end{array}$ \\
\hline \multirow{3}{*}{ Relação com os gastos } & Gasta mais do que ganha & 84 & $16,83 \%$ \\
\hline & Gasta o mesmo que ganha & 239 & $47,90 \%$ \\
\hline & Gasta menos do que ganha & 176 & $35,27 \%$ \\
\hline \multirow{5}{*}{ Frequência que poupa } & Sempre & 45 & $9,02 \%$ \\
\hline & Frequentemente & 173 & $34,67 \%$ \\
\hline & Raramente & 88 & $17,64 \%$ \\
\hline & Às vezes & 163 & $32,67 \%$ \\
\hline & Nunca & 30 & $6,01 \%$ \\
\hline \multirow{4}{*}{$\begin{array}{l}\text { Interação com o dinheiro } \\
\text { anterior a moradia em } \\
\text { Santa Maria }\end{array}$} & Tinha responsabilidade anterior & 198 & $39,68 \%$ \\
\hline & Recebia mesada e controlava & 133 & $26,65 \%$ \\
\hline & Recebia mesada e gastava mais & 23 & $4,61 \%$ \\
\hline & Não tinha preocupações anteriores & 145 & $29,06 \%$ \\
\hline
\end{tabular}

\subsection{ANÁLISE FATORIAL EXPLORATÓRIA}

A fim de aprofundar a compreensão do significado do dinheiro para os estudantes participantes da amostra, foi realizada uma análise fatorial exploratória, com base nas respostas das 82 questões propostas pela Escala de Significado do Dinheiro.

Para proceder a análise fatorial deve-se, inicialmente, medir a adequação das variáveis, por meio do teste KMO e do Teste de esfericidade de Bartlett, para verificar a possibilidade de realizar a técnica que precede a análise fatorial, a ACP (Vicini; Souza, 2005). Para o teste de KMO foi encontrado o valor de 0,818 e para o teste de Bartlett foi 
verificado o valor de $12.847,03$, ambos significativos a $1 \%$, o que indica uma adequabilidade dos dados para a realização da análise de componentes principais.

Para dar continuidade a ACP, foi selecionado, como critério para a extração do número de fatores, o critério da raiz latente, que segundo Hair Jr. et al. (2009), apenas os autovalores maiores que 1 são considerados significantes. Também foram mantidas na análise, as variáveis que apresentaram comunalidade superior a 0,4 (Hair Jr. et al., 2009).

Foram observados 23 autovalores superiores a 1 e que apresentaram comunalidade superior a 0,4 , portando nesta primeira ACP seriam extraídos 23 fatores que explicariam as variáveis originais. Para melhor compreensão e interpretação dos resultados da análise fatorial, optou-se por fixar a quantidade de fatores artificialmente. Para isso, foi realizada a rotação dos fatores, utilizando-se para tal a rotação Varimax normalizada.

Inicialmente, a ACP foi testada com diversos números de fatores fixados, sendo desconsiderados aqueles números de fatores que excluíam uma grande quantidade de variáveis e que não obtinham comunalidade superior a 0,4 , bem como aqueles que não atingiam um percentual satisfatório de variância explicada (VE).

A partir dos resultados obtidos com a ACP, optou-se por manter a fixação de 10 fatores, por apresentar os melhores resultados entre as demais quantidades de fatores testados. Do conjunto inicial das 82 variáveis, foram excluídas 34, por não atenderem ao critério da comunalidade $(>0,4)$.

Após a exclusão das variáveis, foi realizada uma nova verificação da adequabilidade dos dados para a realização da ACP, o que indicou um valor de 0,813 para o teste KMO ede 6631,778 para o teste de esfericidade de Bartlett, indicando a possibilidade da aplicação da ACP, sendo que ambos foram significativos a $1 \%$.

Na Tabela 3 é possível observar os resultados dos autovalores, percentual da variância explicada por cada componente e a variância acumulada.

Tabela 3 - Autovalores e variância explicada

\begin{tabular}{cccc}
\hline \multirow{2}{*}{ Fatores } & Autovalores & \multicolumn{2}{c}{ Variância Explicada } \\
\cline { 3 - 4 } & & Percentual (\%) & Acumulada (\%) \\
\hline 1 & 6,072 & 12,651 & 12,651 \\
2 & 4,382 & 9,128 & 21,779 \\
3 & 2,902 & 6,047 & 27,826 \\
4 & 2,555 & 5,324 & 33,15 \\
5 & 1,768 & 3,684 & 36,834
\end{tabular}




$\begin{array}{cccc}6 & 1,743 & 3,631 & 40,464 \\ 7 & 1,612 & 3,358 & 43,823 \\ 8 & 1,423 & 2,964 & 46,787 \\ 9 & 1,299 & 2,706 & 49,493 \\ 10 & 1,207 & 2,514 & 52,008\end{array}$

Pode-se verificar, na Tabela 3 , que os dois fatores mais importantes que compreendem o significado do dinheiro para os estudantes são o primeiro e o segundo fator, pois explicam, respectivamente, $12,65 \%$ e 9,13\% da variância explicada.

Na Tabela 4 estão expostas as variáveis que compreendem os 10 fatores fixados. Segundo Hair Jr. et al. (2009), a significância das cargas fatoriais de cada fator deve ser de 0,30 para amostras acima de 350 casos. Neste estudo, em que a amostra compreendeu 499 casos, todas as variáveis foram mantidas, pois assumiram cargas fatoriais acima de 0,40 .

O primeiro fator foi composto por 10 variáveis, sendo denominado de Conflito, pois agrupou questões que demonstram que o sofrimento é algo intimamente ligado ao dinheiro, sendo relacionado ao fato de ser capaz de provocar desarmonias, desavenças, ingratidão, dores de cabeça, descontrole emocional, traições, frustrações, jogos de interesse, ilusões e neuroses nas pessoas.

O segundo fator foi denominado de Poder e compreendeu 8 questões que correspondem a sensação de poder causada nas pessoas, pela posse do dinheiro. As variáveis agrupadas neste fator se referem a aspectos como a ascensão social, fatos relacionados ao reconhecimento, a dominação e ao status social que o dinheiro possibilita, bem como a autoridade exercida sobre as demais pessoas e ao prestígio na sociedade obtido pela posse do dinheiro. O terceiro fator, composto por 5 variáveis, foi denominado de Progresso, por abordar questões relacionadas ao desenvolvimento causado na sociedade, pelo uso do dinheiro. Este fator abordou variáveis que inferem que o dinheiro propicia coisas boas para as pessoas, envolvendo questões sobre o fato de que o dinheiro auxilia na evolução da humanidade, garante prosperidade, constrói um mundo melhor, traz esperança para o futuro e facilita a vida das pessoas.

Tabela 4 - Variáveis relacionadas aos fatores

\begin{tabular}{clc}
\hline \multirow{2}{*}{ Fatores } & \multicolumn{1}{c}{ Variáveis } & $\begin{array}{c}\text { Cargas } \\
\text { Fatoriais }\end{array}$ \\
\hline \multirow{3}{*}{ Conflito } & 34- Dinheiro gera desarmonia nas famílias & 0,670 \\
& 37- Dinheiro provoca desavenças com parentes & 0,699 \\
& 38- Dinheiro gera ingratidão & 0,641 \\
& 43- Dinheiro é sinônimo de dor de cabeça & 0,555 \\
\hline
\end{tabular}




\begin{tabular}{|c|c|c|}
\hline & 48- Dinheiro provoca descontrole emocional & 0,670 \\
\hline & 55- Dinheiro provoca traições & 0,564 \\
\hline & 65- Dinheiro provoca frustrações & 0,669 \\
\hline & 68- O dinheiro provoca jogos de interesse & 0,521 \\
\hline & 79- Dinheiro provoca ilusões & 0,590 \\
\hline & 81- Dinheiro provoca neuroses & 0,631 \\
\hline \multirow{8}{*}{ Poder } & 04- Quem tem dinheiro tem autoridade sobre os outros & 0,589 \\
\hline & 06- Dinheiro possibilita ascensão social & 0,593 \\
\hline & 09- Dinheiro traz reconhecimento social & 0,664 \\
\hline & 23- Dinheiro é sinônimo de dominação & 0,561 \\
\hline & 52- Quem tem dinheiro é valorizado socialmente & 0,547 \\
\hline & 59- Ter dinheiro é ter poder & 0,683 \\
\hline & 69- Dinheiro significa status social & 0,679 \\
\hline & 74- É preciso ter dinheiro para ter prestígio & 0,406 \\
\hline \multirow{5}{*}{ Progresso } & 08- O dinheiro constrói um mundo melhor & 0,678 \\
\hline & 18- O dinheiro ajuda a evolução da humanidade & 0,676 \\
\hline & 46- Dinheiro garante prosperidade para a sociedade & 0,715 \\
\hline & 57- Dinheiro traz esperança no futuro & 0,660 \\
\hline & 58- O dinheiro facilita a vida da humanidade & 0,658 \\
\hline \multirow{5}{*}{ Prazer } & 11- Dinheiro significa prazer & 0,493 \\
\hline & 16- O dinheiro ajuda a tornar as relações amorosas mais agradáveis & 0,551 \\
\hline & 44- Dinheiro atrai felicidade & 0,536 \\
\hline & 47- O dinheiro facilita a convivência familiar & 0,776 \\
\hline & 82- Dinheiro ajuda a ter harmonia familiar & 0,762 \\
\hline \multirow{5}{*}{$\begin{array}{c}\text { Cultura e } \\
\text { Desenvolvimento }\end{array}$} & 12- Com dinheiro eu patrocinaria o desenvolvimento das artes & 0,577 \\
\hline & 35- Com dinheiro eu investiria em pesquisas científicas & 0,724 \\
\hline & 56- Quem tem dinheiro deve empregá-lo no desenvolvimento do país & 0,512 \\
\hline & 63- Eu investiria dinheiro em eventos culturais & 0,697 \\
\hline & 78- Eu investiria dinheiro em inovações tecnológicas & 0,691 \\
\hline \multirow{5}{*}{ Poder Negativo } & 26- Crianças ricas são ensinadas a evitar contato com crianças pobres & 0,636 \\
\hline & 31- Quem tem dinheiro é o primeiro a ser atendido em todos os lugares & 0,677 \\
\hline & 40- As pessoas submetem-se a quem tem dinheiro & 0,466 \\
\hline & 66- Quem tem dinheiro passa por cima das normas estabelecidas & 0,522 \\
\hline & 77- Crianças ricas são educadas para mandar & 0,609 \\
\hline \multirow{4}{*}{ Espiritualidade } & 05- Quem tem fé sabe que precisa fazer caridade & 0,681 \\
\hline & 29- Recompensas espirituais são mais importantes que dinheiro & 0,467 \\
\hline & 60- É dever de todas as pessoas dividirem o que têm & 0,469 \\
\hline & 67- Basta crer em Deus para ter as necessidades atendidas & 0,667 \\
\hline \multirow{2}{*}{ Sofrimento } & 24- Dinheiro é uma coisa complicada para mim & 0,663 \\
\hline & 32- Pensar em dinheiro me deixa deprimido & 0,673 \\
\hline \multirow{2}{*}{ Generosidade } & 49- Ajudar quem precisa é melhor que guardar dinheiro & 0,683 \\
\hline & 50- Os pais devem ensinar os filhos a serem generosos & 0,776 \\
\hline \multirow{2}{*}{ Saúde } & 13- Acho importante ter seguro de vida & 0,727 \\
\hline & 54- Acho importante fazer convênios de saúde & 0,756 \\
\hline
\end{tabular}

O quarto fator foi composto por 5 questões que estão relacionadas a visão positiva do dinheiro sendo, por isso, denominado de Prazer. Neste fator permaneceram questões sobre os benefícios trazidos pelo dinheiro em relação ao convívio familiar e amoroso, bem como sobre a felicidade e a harmonia por ele gerada. 
O quinto fator foi denominado de Cultura e Desenvolvimento, pois apresenta 5 afirmativas que abordam sobre a atitude de investir em pesquisas científicas, cultura, artes e inovação tecnológica.

O fator de número seis, composto por 5 questões, foi renomeado de Poder Negativo, pois aborda variáveis que representam aspectos ruins a respeito do dinheiro. As questões se referem a situações preconceituosas que o dinheiro pode causar, a ações desiguais que acabam favorecendo as pessoas mais ricas, bem como a forma como este pode ser usado para a manipulação das pessoas e das normas. Este fator aborda a imagem fortemente negativa que as pessoas associam ao dinheiro.

O sétimo e oitavo fator foram nomeados, respectivamente, de Espiritualidade, contendo 4 questões, e de Sofrimento, contendo 2 variáveis. O fator Espiritualidade compreendeu questões relacionadas à fé das pessoas, ou seja, a crença em Deus e a necessidade de ajudar ao próximo, enquanto o fator Sofrimento abordou variáveis que representam uma exposição das amarguras trazidas pelo dinheiro. O nono fator, Generosidade, compreendeu 2 questões sobre o fato de ajudar ao próximo e ser generoso. Por fim, o décimo fator é o resultado de 2 questões que abordam a segurança que o dinheiro é capaz de gerar em se tratando de saúde das pessoas e, por este motivo, recebeu o nome de Saúde.

Na Tabela 5podem ser observados os valores dos Alfas de Cronbach obtidos a partir da amostra estudada. Valores entre 0,6 e 0,7 são aceitáveis, sendo que os valores abaixo disto indicam uma escala não confiável. Porém, para alguns construtos psicológicos, valores abaixo de 0,6 podem ser esperados devido à diversidade do que está sendo medido (Hair Jr. et al., 2005; Malhotra, 2006; Field, 2009;).

Na Tabela 5 podem ser observados os valores de alfa de Cronbach encontrados para cada um dos fatores. Também é possível verificar os valores dos Alfas de cada construto caso alguma variável fosse excluída, o que permitiu inferir que esta ação levaria a um resultado menos satisfatório.

Os valores dos Alfas de Cronbach dos fatores de 1 a 6 mostraram-se satisfatórios, pois assumiram valores acima de 0,6.

Tabela 5 - Alfas de Cronbach para cada fator

\begin{tabular}{|c|c|c|c|c|c|}
\hline \multirow{2}{*}{ Fatores } & \multirow{2}{*}{$\alpha$} & \multicolumn{3}{|c|}{$\alpha$ se a variável for excluída } & \multirow[b]{2}{*}{$\alpha$} \\
\hline & & Variável & $\alpha$ & Variável & \\
\hline 1 - Conflito (10 itens) & 0,85 & Q34 & 0,83 & Q55 & 0,83 \\
\hline
\end{tabular}




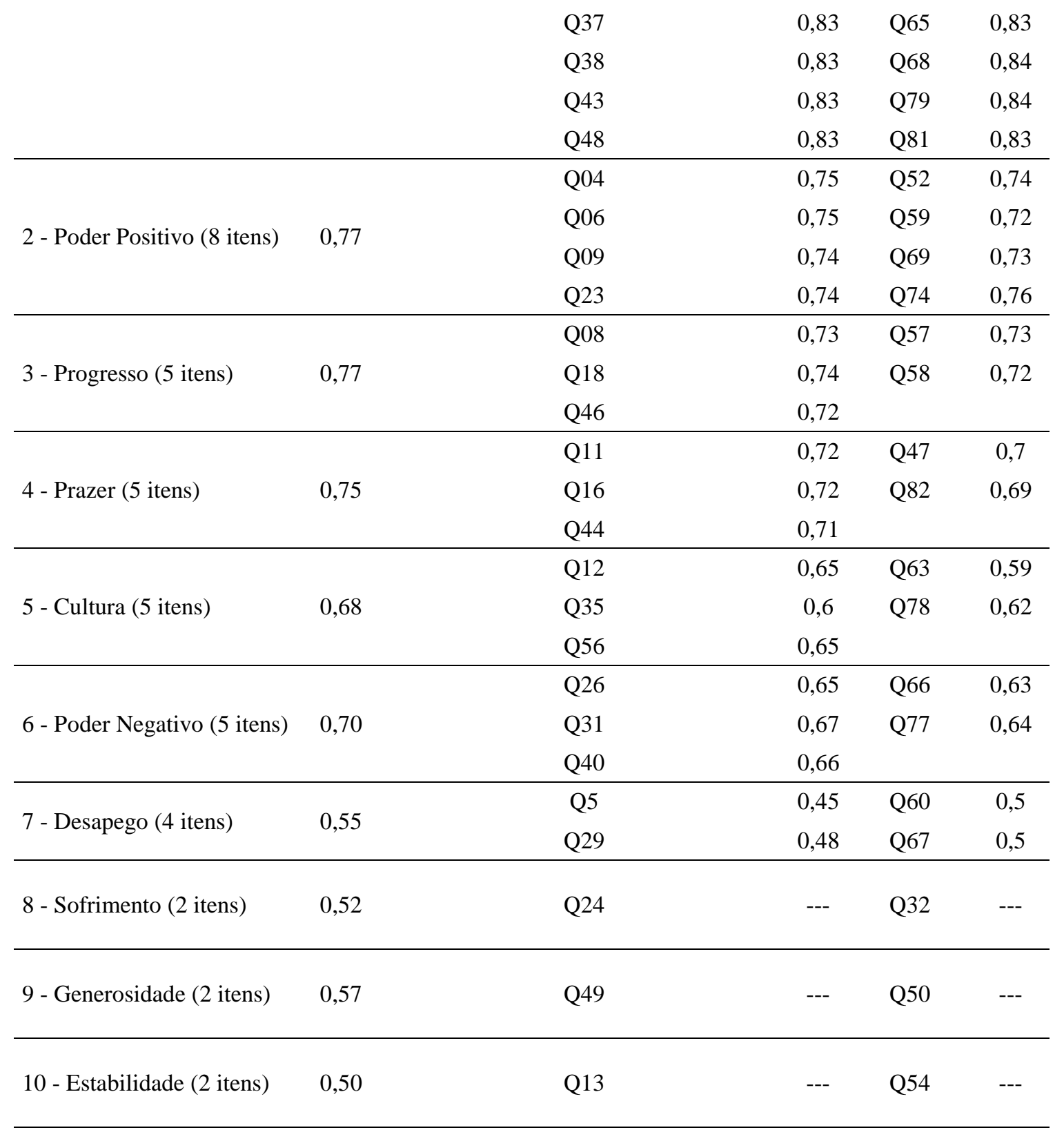

Observando-se os valores dos Alfas de Cronbach, foi possível verificar que os alfas dos fatores de 7 a 10 possuem coeficientes inferiores a 0,6 , o que pode ser devido ao número pequeno de questões que foram utilizadas para compor estes fatores. $\mathrm{O}$ alfa de Cronbach geral da Escala de Significado do Dinheiro foi satisfatório, com valor de 0,813 .

\section{CONSIDERAÇÕES FINAIS}

Nesta pesquisa foram abordados aspectos da psicologia econômica que consiste em estudar o comportamento financeiro dos indivíduos, tendo como variáveis, 
pensamentos, sentimentos, crenças, expectativas, entre outras. Um dos comportamentos mais analisados pelos estudos dessa área, tem sido relacionado ao significado do dinheiro. Em essência, as pesquisas que abordam os mais diversos assuntos relacionados ao dinheiro, buscam entender o perfil psicológico, principalmente do ponto de vista comportamental dos indivíduos.

Neste contexto, o presente estudo teve como objetivo investigar qual o significado do dinheiro para jovens universitários da UFSM. Analisando-se o perfil dos respondentes, foi possível verificar que a média de idade dos estudantes é de 21 anos, 92,38\% residiam em municípios do Estado do Rio Grande do Sul antes de se mudarem para Santa Maria, 96,59\% são solteiros, 98,80\% não possuem filhos e 42,28\% moram em lugares alugados. A maioria dos respondentes gasta o mesmo valor que ganha $(47,90 \%)$, costuma poupar frequentemente $(34,67 \%)$ e já possuíam responsabilidades com o dinheiro antes de se mudarem $(39,68 \%)$.

A fim de identificar, por meio da formação de fatores, o significado do dinheiro para os estudantes, foi realizada uma análise fatorial exploratória. Esta técnica multivariada permitiu expressar, o conjunto inicial de 82 variáveis, em apenas 10 fatores, compostos por 48 questões que permaneceram na análise.

Os dois fatores mais relevantes, o primeiro denominado de Conflito (VE = $12,65 \%)$ e o segundo de Poder ( $\mathrm{VE}=9,12 \%)$, compreenderam variáveis que abordam questões relacionadas aos problemas que o dinheiro pode causar, como desavenças, desarmonia, traições e frustações, bem como ao poder e privilégio social associado a posse do dinheiro como, por exemplo, a ascensão social que este permite, a autoridade, a dominação e o prestígio. Os demais fatores, foram denominados de: Progresso (VE = 6,04\%), Prazer (5,32\%), Cultura e Desenvolvimento (3,68\%), Poder Negativo (VE = $3,63 \%)$, Espiritualidade ( $\mathrm{VE}=3,35 \%)$, Sofrimento $(2,96 \%)$, Generosidade $(2,71 \%)$ e Saúde $(2,51 \%)$.

Em suma, acredita-se que o dinheiro possui significados que vão muito além de sua função principal, que seria a função de troca, ou seja, que existem outros fatores associados ao dinheiro para os estudantes respondentes, como o poder, a autoridade, a ascensão social, o prazer, a generosidade, os cuidados com a saúde, a importância do desenvolvimento social, entre outras características inerentes a esse grupo de indivíduos.

Como sugestão de trabalhos futuros, sugere-se a realização de um estudo que analise o significado do valor do dinheiro comparando cursos universitários, classes 
sociais e faixas etárias dos entrevistados. Também sugere-se expandir esta pesquisa para alunos de pós-graduação. Assim como, a realização desta mesma pesquisa não somente de maneira quantitativa, mas também qualitativa, realizando-se entrevistas com os estudantes.

\section{REFERÊNCIAS}

ARRUDA, P. B. Uma investigação sobre o efeito disposição. Dissertação (Mestrado em Engenharia de Produção) - Universidade Federal de Santa Catarina, Florianópolis, 2006.

BONDT, W. F. M; THALER, R. Does the stock market overreact?. The Journal of finance, v. 40, n. 3, p. 793-805, 1985.

FIELD, A. Descobrindo a estatística usando o SPSS. 2 . d. Porto Alegre: Artmed, 2009.

HAIR, Jr. J. F.; ANDERSON, R. E.; TATHAM, R. L.; BLACK, W. Fundamentos de Métodos de Pesquisa em Administração. Porto Alegre: Bookman, 2005.

HALFELD, M.; TORRES, F. F. L. Finanças Comportamentais: aplicações no contexto brasileiro. RAE - Revista de Administração de Empresas. v. 41, n. 2, p. 64-71. Abr./Jun. 2001.

LOEWENSTEIN, G. Out of control: Visceral influences on behavior. Organizational Behaviorand Human Decision Processes, v. 3, n. 65, pp. 272-292, 1996.

MACEDO, J. S. Teoria do prospecto: uma investigação utilizando simulação de investimentos. Tese (Doutorado em Engenharia de Produção) - Curso de Pós-graduação em Engenharia de Produção, Universidade Federal de Santa Catarina, 2003.

MALHOTRA; N. K. Pesquisa de marketing: uma orientação aplicada. 3. ed. Porto Alegre, Bookman, p. 720, 2001.

Pesquisa de Marketing: uma orientação aplicada. Tradução Laura Bocco. 4. ed. Porto Alegre: Bookman, 2006.

MOREIRA, A. S. Valores e dinheiros: um estudo transcultural da relação entre prioridades de valores e significado do dinheiro para indivíduos. Tese de doutorado. Universidade de Brasília, Brasília, 2000.

NOFSINGER, J. R. A Lógica do Mercado. Fundamento: São Paulo, 2006.

PESTANA, M.; GAGEIRO, J. Análise de dados para ciências sociais: a complementaridade do SPSS. Lisboa: Edições Silabo, 2003. 
SCHWARTZ, S. H. Universals in the content and structure of human values: theoretical advances and empirical tests in 20 countries. Advances in experimental social psychology. Orlando, F. L. Academic, v. 25, p. 1-65, 1992.

SIMON, H. A. Administrative behavior. Totawa, NJ: Littlefield, Adams, 2. ed. 1957.

SIMON, H. Comportamento Administrativo: estudo do processo decisório nas organizações administrativas. Rio de Janeiro: FGV, 1979.

SORTINO, F. A. From alpha toomega. In SORTINO, F. A., \& SATCHELL, S., (org). Managing down side risk in financial markets: Theory, practice and implementation (pp. 3-25). Buttworth Heinemann: Oxford, 2001.

TVERSKY, A.; KAHNEMAN, D. Judgment under Uncertainty: Heuristics and Biases. Science, Set/1974.

TVERSKY, A.; KAHNEMAN, D. Prospect theory: an analysis of decision under risk. Econometrics, New York, v. 4, n. 2, p. 263-291, 1979.

VERGARA, S. C. Projetos e relatórios de pesquisa em administração. 3. ed. São Paulo: Atlas, 2000.

VICINI, L.; SOUZA, A. M. Análise multivariada da teoria à prática. Santa Maria: UFSM, CCNE, 2005.

ZERRENNER, S. A. Estudo sobre as razões para a população de baixa renda. 2007. (Dissertação de Mestrado em Ciências Administrativas) - Universidade de São Paulo, São Paulo, 2007. 